\title{
Advanced carcinoma of the prostate - does the pre-treatment Leydig cell function determine the response to orchidectomy?
}

\author{
A. L. Houghton* \\ M.B., F.R.C.S. \\ H. S. JACOBS $\dagger$ \\ M.D., M.R.C.P. \\ *Department of Pathology and Cancer Research, University of Leeds, Leeds 1, and \\ $\dagger$ †epartment of Obstetrics and Gynaecology, St Mary's Hospital Medical School, London
}

\begin{abstract}
Summary
In a study of twenty patients there was no support for the hypothesis that the response to orchidectomy of patients with advanced prostatic cancer depends on pre-operative Leydig cell function. The only significant prognostic factor was the histological grade of the primary tumour.
\end{abstract}

\section{Introduction}

Bilateral orchidectomy has been a standard form of treatment for advanced prostatic cancer since Huggins and his colleagues in 1941 showed that many patients with the disease experienced clinical improvement, with osteosclerosis of metastases three to six months after castration. Huggins et al. noted that the presence of testes markedly lighter than the normal weight was a bad prognostic sign and that the clinical course following orchidectomy could also be related to the cellular pattern of the primary tumour, being unfavourable when the tumour was undifferentiated (Huggins, 1942; Huggins and Hodges, 1941 ; Huggins, Stevens and Hodges, 1941).

The efficacy of castration has been ascribed to a reduction in testicular hormones and measurement of plasma testosterone levels before and after both total and subcapsular orchidectomy has confirmed that considerable reduction of testosterone levels occurs (Robinson and Thomas, 1971; Shearer et al., 1973; Burge et al., 1976). These and other observations have led to the view that carcinoma of the prostate is an androgen-dependent tumour, despite the paradox that the condition occurs predominantly in men past the fifth decade at a time when testicular function is often declining (Leading Article, 1975). Moreover no significant differences in the levels of plasma testosterone between men with carcinoma of the prostate and age-matched controls have been reported, although the levels may be lower in men with metastatic carcinoma of the prostate (Robinson and Thomas, 1971).

Not all patients with advanced prostatic cancer respond favourably to castration. In a preliminary

Requests for reprints to Mr A. L. Houghton. study of three elderly patients undergoing bilateral subcapsular orchidectomy for advanced prostatic cancer, Bramble and Jacobs (1975) found that preoperative testicular function, as reflected by the plasma concentrations of testosterone, luteinising hormone (LH) and follicle-stimulating hormone (FSH), varied widely between the values found in young men and those observed after castration. Since the magnitude of the changes induced by castration clearly depend on pre-operative testicular function, Bramble and Jacobs questioned whether this factor determines the response to orchidectomy of patients with advanced prostatic cancer. The purpose of the present study was to test this hypothesis.

\section{Patients and methods}

The study was performed on twenty patients suffering from advanced prostatic cancer - Veterans Stage 3 and 4 (VACURG, 1967). They were assessed before and six months after bilateral orchidectomy according to the European Organization for Research on Treatment of Cancer (EORTC) protocol No. 30740. The histology of the prostate was graded according to the Union Internationale contre le Cancer (1974).

\section{Hormone measurements}

Blood samples were obtained before operation and six months later. The plasma was separated and stored at $-20^{\circ} \mathrm{C}$ until assayed. The pre- and postoperative samples of every patient were measured in the same assay. Plasma testosterone was determined by radio-immuno assay (RIA) in the Division of Steroid Endocrinology, University of Leeds, using a testosterone antiserum raised in a rabbit against testosterone - 3 - $(O$ - carboxymethyl $)$ - oxime - bovine serum albumin conjugate prepared according to the method described by Habib et al. (1976). The normal range in men aged $18-60$ years is $8-27 \mathrm{nmol} / \mathrm{l}$. Since circulating dihydrotestosterone (DHT) levels are about one-tenth those of testosterone in adult 
men aged 20-39 years, rising to about one-fifth in men aged 60-90 years (Horton et al., 1975), and the cross reactivity with DHT of the antiserum used was $52 \%$, no correction was applied for any DHT present. Plasma LH and FSH were measured in specific RIA, using the method described by Jacobs and Lawton (1974). Standard LH (MRC 68/40) and FSH (MRC 68/39) were provided by the National Institute for Biological Standards and Control of the Medical Research Council of the UK. The normal ranges for men aged 18-40 years are:

$$
\begin{aligned}
& 0.2-2.0 \mathrm{ng} / \mathrm{ml} \mathrm{LH} \text { and } \\
& 16-50 \mathrm{ng} / \mathrm{ml} \mathrm{FSH}
\end{aligned}
$$

\section{Treatment}

Apart from bilateral orchidectomy (total or subcapsular), the only additional treatments were transurethral resection of the prostate and analgesics.

\section{Assessment}

Clinical assessment was made at six months. The most important subjective parameters used were improvement in performance status and reduction in pain. The most important indications of objective remissions used were diminution of the number or size of metastases, or a $50 \%$ reduction in the size of the primary tumour, followed by reduction in the serum acid phosphatase, rise in haemoglobin and

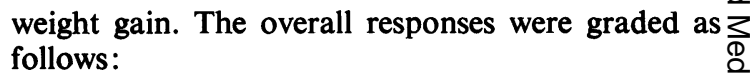

(1) Very good - subjective and objective remission.. .

(2) Good - subjective remission.

(3) No change.

(4) Poor - progression of disease.

\section{Results}

Table 1 shows the age, stage, histological grade, $\stackrel{\triangleright}{\unrhd}$ type of orchidectomy, pre-orchidectomy concen-» trations of FSH, LH and testosterone and response $-\overrightarrow{0}$ to treatment. Fig. 1 shows the pre- and the 6-month post-orchidectomy concentrations of FSH, LH and $\vec{\omega}$ testosterone.

Fig. 2 shows the pre-treatment levels of FSH, LH윽 and testosterone in relation to response. Clinical 3 . improvement was seen in $50 \%$ of the patients at $\perp$ 6 months.

\section{Discussion}

There were no significant differences with respect to age, clinical stage, type of operation or pre- $]$ treatment levels of FSH, LH and testosterone be- $\vec{\rightarrow}$ tween the groups of patients that were improved oro not improved after castration. There was no significant difference between the fall of plasna $\vec{\varphi}$ testosterone with respect to the type of operationos performed. Pre-operatively two of the patients hodic 'compensated' Leydig cell failure, as indicated

TABLE 1. Details in twenty patients evaluated 6 months after orchidectomy

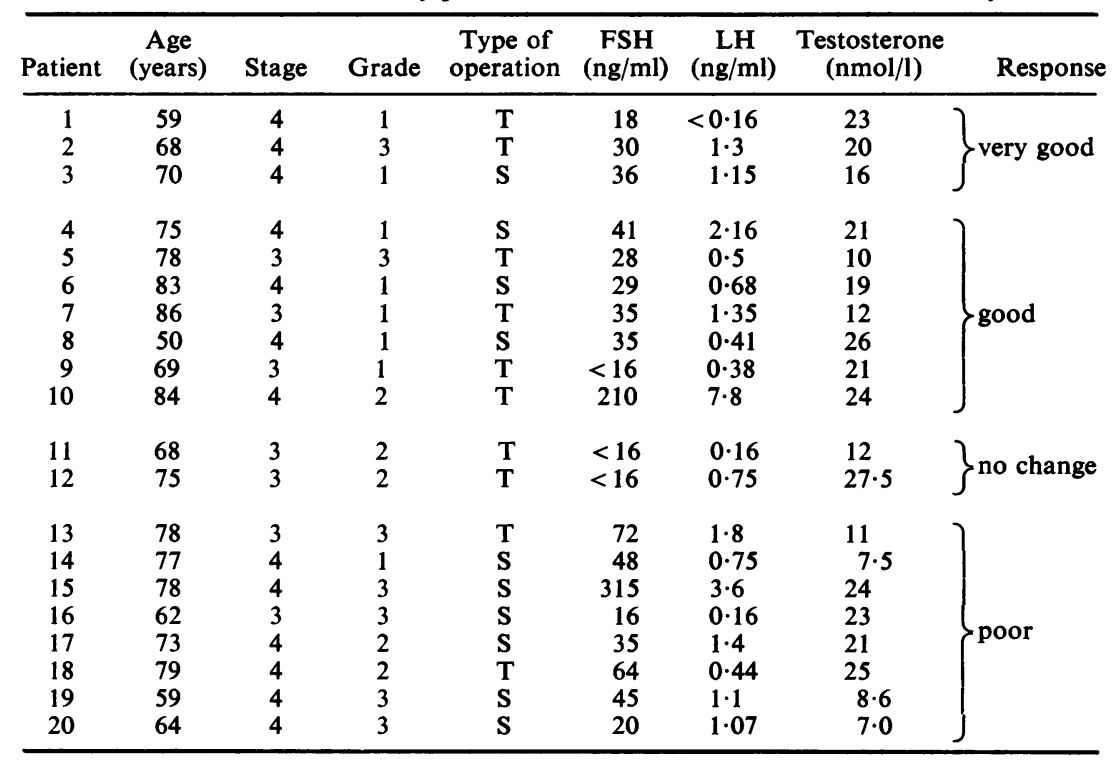

$\mathbf{T}=$ Total orchidectomy.

$\mathbf{S}=$ Subcapsular orchidectomy. 

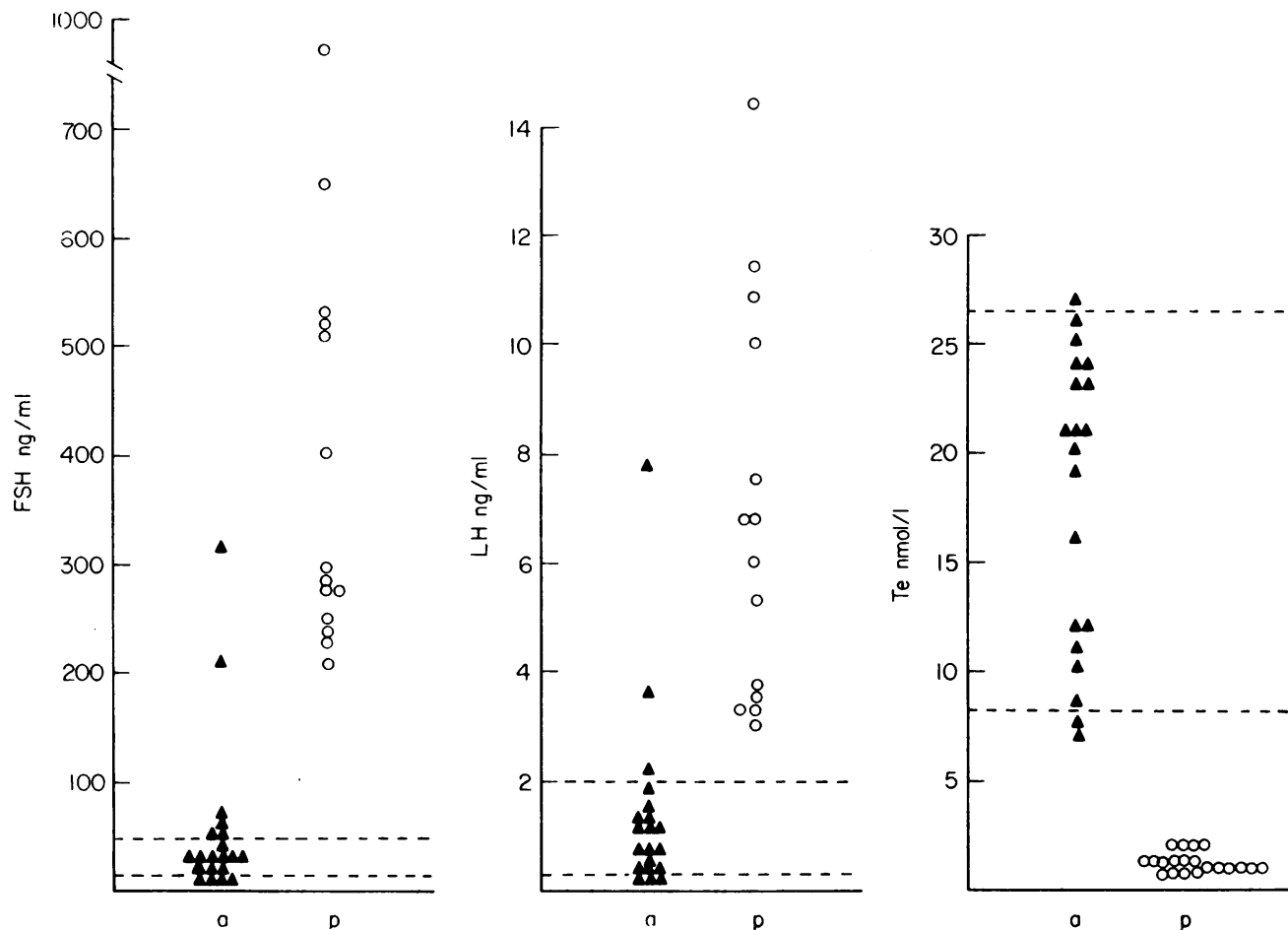

Fig. 1. Serum levels of FSH, LH and testosterone. (a) pre-orchidectomy (closed triangle) and (p) postorchidectomy (open circles); dotted lines represent upper and lower limits of normality (see text).
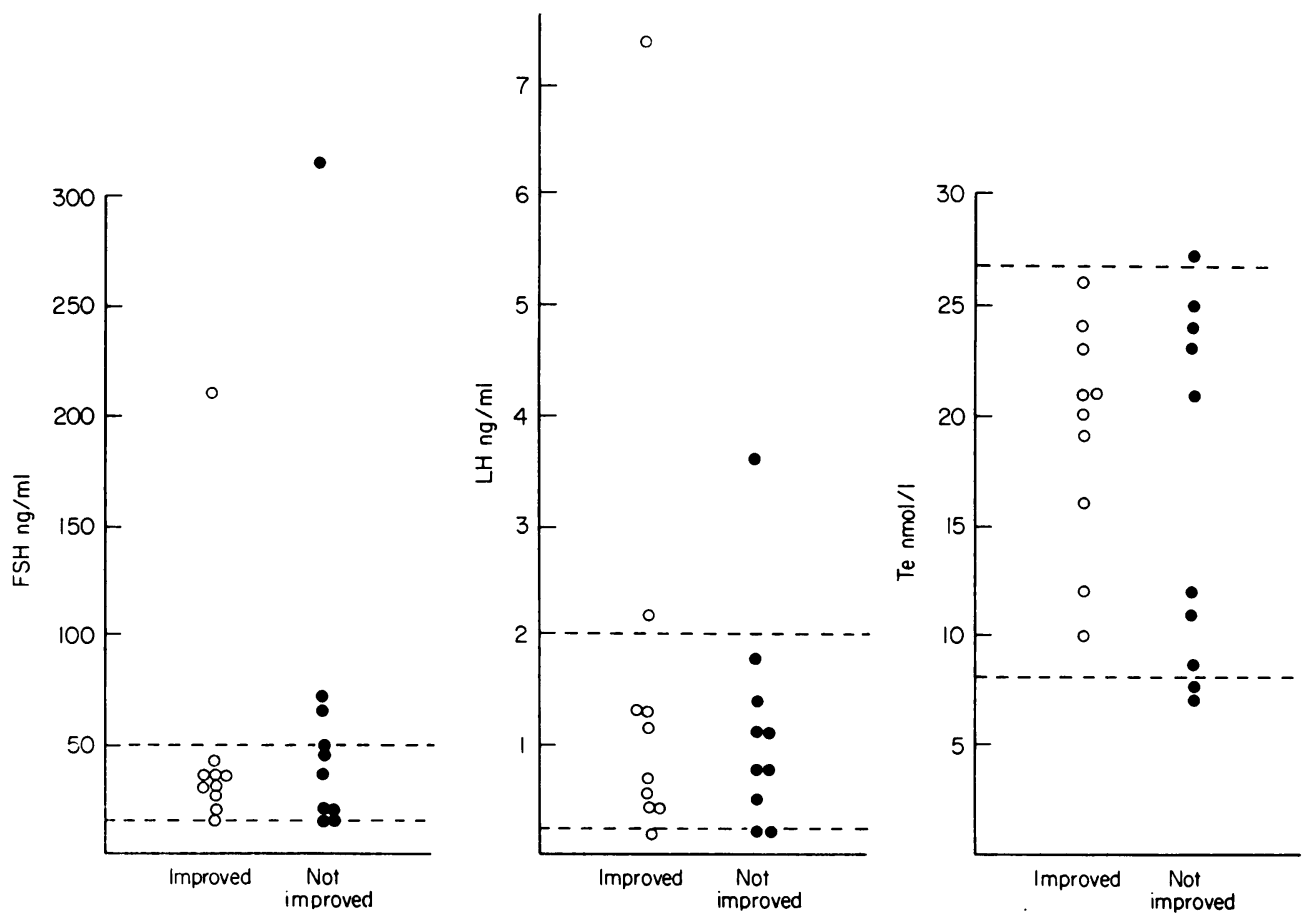

FIG. 2. Pre-orchidectomy serum levels of FSH, LH and testosterone in relation to clinical response. Open circles represent patients showing 'improvement'. Closed circles represent patients showing 'no improvement'; dotted lines represent upper and lower limits of normality (see text). 
high LH but normal testosterone levels (Bramble et al., 1975). Both these patients also had high levels of FSH.

\begin{tabular}{lcc}
\hline TABLE 2. Clinical response and tumour \\
grading
\end{tabular}

It is not unexpected for patients in this age group to have elevated levels of $\mathrm{LH}$ and FSH (Baier, Biro and Weinges, 1974; Stearns et al., 1974) but it is clear that it is of no prognostic importance with respect to treatment by orchidectomy. These measurements are analagous to Huggins' measurement of testicular weight and it is interesting to note that the three patients of Huggins (1942) who had small testes also had tumours that were undifferentiated. It is perhaps this latter factor that was more important from the prognostic point of view, particularly as in the present series seven of the ten patients showing improvement had well differentiated tumours, while only one of the ten who did not improve had a well differentiated tumour (Table 2). This difference was statistically significant (Fisher's Exact Test $P=0.05$ ). These data therefore confirm Huggins' original (1942) observations on the prognostic importance of the histological grade of the primary tumour and suggest that pre-operative Leydig cell function should not be used to determine the need for endocrine therapy.

\section{Acknowledgments}

Mr. A. L. Houghton is supported by the Yorkshire Regional Cancer Organization.

The Surgeons of the Yorkshire Urological Group, Mr P. B. Clark, Mr R. W. Glashan, Mr M. R. G. Robinson, Mr P. H. Smith and Mr R. E. Williams, for encouragement and allowing us to study their patients.

Mrs M. Cawood, Department of Steroid Endocrinology, University of Leeds, for advice on testosterone measurement.

Dr M. K. Mason, Consultant Pathologist, St James's Hospital, Leeds, for reviewing the histological slides.

\section{References}

Baier, H., Biro, G. \& Weinges, K.F. (1974) Serum levels o FSH, LH and testosterone in human males. Hormone and Metabolic Research, 6, 514.

Bramble, F.J., Houghton, A.L., Eccles, S.S., Murray M.A.F. \& JACOBS, H.S. (1975) Compensated hypogonad: ism in patients with primary testicular disorders: effects of human chorionic gonadotrophin. Journal of Endocrinology 65, 10.

BRAMBle, F.J. \& JACOBS, H.S. (1975) Hormones, elderly testes and carcinoma of the prostate. British Medica? Journal, 3, 307.

Burge, P.D., Harper, M.E., Hartog, M. \& Gingell, J.C. (1976) Subcapsular orchidectomy - an effective operation? Proceedings of the Royal Society of Medicine, 69, 663.

Habib, F.K., Lee, I.R., Stitch, S.R. \& SMith, P.H. (1976) Androgen levels in the plasma and prostatic tissues of patients with benign hypertrophy and carcinoma of theo prostate. Journal of Endocrinology, 71, 99.

Horton, R., Hsieh, P., Barberia, J., Pages, L. \& CosgroveciM. (1975) Altered blood androgens in elderly men with ${ }^{+}$ prostatic hyperplasia. Journal of Clinical Endocrinology and
Metabolism, 41, 793.

HugGINS, C. (1942) Effect of orchidectomy and irradiation on cancer of the prostate. Annals of Surgery, 115, 1192. 오

HugGins, C. \& Hodges, C.V. (1941) Studies on prostatico cancer. I. The effect of castration, of estrogen and of androgen injection on serum phosphatases in metastatic carcinoma of the prostate. Cancer Research, 1, 293.

Huggins, C., Stevens, R.E. \& Hodges, C.V. (1941) Studies on prostatic cancer. II. The effects of castration on ad- $\overrightarrow{-}$ vanced carcinoma of the prostate gland. Archives $8 f$ Surgery, 43, 209.

JACOBS, H.S. \& LAwTON, N.F. (1974) Pituitary and placent glycopeptide hormones. British Medical Bulletin, 30, 5s은

LEADING ARTICLE (1975) Hormones and elderly testes. British Medical Journal, 3, 2.

Robinson, M.R.G. \& Thomas, B.S. (1971) Effect of hor-ฏू monal therapy on plasma testosterone levels in prostatic $\frac{0}{\mathbb{Q}}$ carcinoma. British Medical Journal, 4, 391.

Scientific Tables. Documenta Giegy. 6th edn, p. 109.

Shearer, R.J., Hendry, W.F., Sommerville, I.F. \& Fer-oํㅡㄹ GUSSON, J.D. (1973) Plasma testosterone: an accurate monitor of hormone treatment in prostatic cancer. British Journal of Urology, 45, 668.

Stearns, E.L., MacDonnell, J.A., Kaufman, B.J., Padua R., LuCMAN, T.S., Winter, J.S.D. \& Faiman, C. (1974) Declining testicular function with age. Hormonal and clinical correlates. American Journal of Medicine, 57, 761.

UNION INTERNATIONALE CONTRE LE CANCER (1974) TNM: Classification of Malignant Tumours, 2nd edn, pp. 79-82. Geneva.

Veterans Administration Cooperative Urological Re-응 SEARCH GROUP (1967) Treatment and survival of patients with cancer of the prostate. Surgery, Gynecology and $\frac{D}{O}$ Obstetrics, 124 (2), 1011. 